\title{
Multisymplectic structures and the variational bicomplex
}

\author{
Thomas J. Bridges, Peter E. Hydon ${ }^{\dagger}$ \\ Department of Mathematics \\ University of Surrey, Guildford, Surrey, GU2 7XH, UK \\ Jeffrey K. Lawson \\ Department of Mathematics and Computer Science \\ Western Carolina University, Cullowhee, NC 28723, USA
}

June 1, 2009

\begin{abstract}
Multisymplecticity and the variational bicomplex are two subjects which have developed independently. Our main observation is that re-analysis of multisymplectic systems from the view of the variational bicomplex not only is natural but also generates new fundamental ideas about multisymplectic Hamiltonian PDEs. The variational bicomplex provides a natural grading of differential forms according to their base and fibre components, and this structure generates a new relation between the geometry of the base, covariant multisymplectic PDEs and the conservation of symplecticity. Our formulation also suggests a new view of Noether theory for multisymplectic systems, leading to a definition of multimomentum maps that we apply to give a coordinate-free description of multisymplectic relative equilibria. Our principal example is the class of multisymplectic systems on the total exterior algebra bundle over a Riemannian manifold.
\end{abstract}

\footnotetext{
${ }^{*}$ t. bridges@surrey.ac.uk

${ }^{\dagger}$ p.hydon@surrey.ac.uk

${ }^{\ddagger}$ jlawson@wcu.edu
} 


\section{Introduction}

One of the great triumphs of symplectic geometry was the realization that the concept of a Hamiltonian system could be deduced from the properties of a manifold hosting a nondegenerate closed two-form. A smooth even-dimensional manifold $P$ equipped with a closed nondegenerate two-form $\omega$ is a symplectic manifold; diffeomorphisms on $P$ that preserve $\omega$ are Hamiltonian flows. This latter observation follows from Cartan's formula: by differentiating $\varphi^{*} \omega=\omega$, where $\varphi$ is a diffeomorphism on $P$ generated by the vector field $\mathbf{v}$, one obtains

$$
\left.\left.0=\mathcal{L}_{\mathbf{v}} \omega=\mathbf{v}\right\lrcorner \mathrm{d} \omega+\mathrm{d}(\mathbf{v}\lrcorner \omega\right)
$$

Closure of $\omega$ then implies $\mathrm{d}(\mathbf{v}\rfloor \omega)=0$ and so (locally) there exists a function $H: P \rightarrow \mathbb{R}$ with

$$
\mathbf{v} \downarrow \omega=-\mathrm{d} H
$$

recovering the classical definition of a Hamiltonian system. On the other hand, if $\varphi$ is generated by a Hamiltonian function then the above argument in reverse shows that $\varphi^{*} \omega=\omega$.

Given any smooth manifold $Q$, there is a canonical closed nondegenerate two-form on $T^{*} Q$. In addition to showing that Hamiltonian systems are plentiful, these observations provide an abstract class of Hamiltonian ordinary differential equations (ODEs) for which general theorems and methodologies can be deduced. In other words, one does not need to study each specific Hamiltonian system that arises in applications; instead, one can obtain general results that apply to the class of Hamiltonian systems on a cotangent bundle, (cf. Chapter 6 of MARSDEN \& RATiU [19], for example).

This approach contrasts with the historical view whereby Hamiltonian systems arise from the Legendre transform of a given nondegenerate Lagrangian. The Legendre transform gives rise to a natural class of Hamiltonian systems on the cotangent bundle of the configuration space. A general theory for Hamiltonian systems on cotangent bundles will include the class generated by the Legendre transform. But the abstract class of Hamiltonian systems on $\left(T^{*} Q, \omega\right)$ is much larger, and that on $(P, \omega)$ is larger still.

How should these ideas be generalized for partial differential equations (PDEs)? One of the motivations for this question is that the Legendre transform for PDEs is not always - indeed rarely - well behaved. Therefore, we are interested in the class of PDEs for which the analogue of (1.2) can be developed systematically.

Fibre bundles provide the natural language for dealing with such PDEs. Let $E=X \times U$, where $X$ is the base manifold (that is, the space of independent variables) and $U$ is the space of dependent variables (fields). Solutions of PDEs can be characterized as sections of the bundle $E$. For simplicity, we restrict attention to trivial bundles over an oriented Riemannian manifold $X$, but some results apply more generally. Our analysis will be predominantly local, so we fix a chart and take local coordinates $x^{i}$. The tangent space at each $x \in X$ is $T_{x} X$, with a canonical basis $\left\{\frac{\partial}{\partial x^{i}}\right\}$. The cotangent space $T_{x}^{*} X$ has a canonical basis $\left\{\mathrm{d} x^{i}\right\}$; differential $k$-forms map $X$ to the exterior algebra of $T_{x}^{*} X$. The covariant metric $g_{x}: T_{x} X \times T_{x} X \rightarrow \mathbb{R}$ has coefficients

$g_{i j}=g_{x}\left(\frac{\partial}{\partial x^{i}}, \frac{\partial}{\partial x^{j}}\right)$; the contravariant metric $g_{x}^{*}: T_{x}^{*} X \times T_{x}^{*} X \rightarrow \mathbb{R}$ induces a metric on the space of differential $k$-forms (which is used in $\S 5$ ).

To determine the appropriate class of manifolds that generalize the cotangent bundle construction to PDEs, the Legendre transform should provide a clue. In the ODE case, the 
Legendre transform leads to Hamiltonian ODEs on the cotangent bundle of the configuration space. For PDEs generated by a nondegenerate Lagrangian (such as a nondegenerate first-order field theory), the Legendre transform leads to an affine bundle modelled on the vector bundle of linear maps from TU to TX (cf. GotAY ET AL. [13] and references therein). However the Cartan form, which generalizes the symplectic form, is determined by the Lagrangian and not merely by the manifold; the Hamiltonian functional in this construction appears as part of the Cartan form.

In order to bypass the Lagrangian, CANTRIJn ET AL. [10] extend the concept of a "multisymplectic manifold" (a concept attributed to Tulczyjew - cf. BINZ ET AL. [5]) to mean an $n$-dimensional manifold with with a closed nondegenerate $(k+1)$-form where $1<k+1 \leq n$. (The case $k=1$ recovers the definition of symplectic manifold.) In the case of field theory, $k$ is the dimension of the base manifold. Taking this manifold as a starting point, a number of interesting differential geometric results can be deduced [10], and this construction would seem to lead to a natural generalization of (1.1). However, contracting a $(k+1)$-form with a vector field produces a $k$-form, and unless $k=1$, the Hamiltonian function is replaced by a tensor-valued field, which is not typical in applications. This problem is partially due to the fact that this approach offers no natural distinction between base coordinates and fibre coordinates, so there is no obvious way to construct sections as models for the solution space of PDEs. To model PDEs, a Lagrangian is used to organize the coordinates, leading to constructions based on the Cartan form. Kanatchikov [15] and PAufler \& Römer [22] propose a formula generalizing (1.2), namely

$$
\mathbf{v} \_\omega=\mathrm{d} f,
$$

where $\omega$ is a closed nondegenerate $(n+1)$-form (a variant of the Cartan form) and $n$ is the dimension of the base manifold. However, $f$ is an $r$-form and $\mathbf{v}$ is a multivector field of tensor grade $(n-r)$.

The $k$-symplectic geometry of NoRRIS [21] comes closest to a generalization of (1.2) based on the geometry of the manifold only (see also LAWSON [16], DE LEON ET AL. [18] and references therein). Given any smooth manifold, $k$-symplectic geometry is based on the geometry of the frame bundle and generalizes the canonical symplectic structure on the cotangent bundle of a manifold. However, this structure also generates a tensor-valued Hamiltonian function and an unconventional class of Hamiltonian PDEs.

In this paper, a new approach to this problem is proposed. We put greater emphasis on the base manifold and we use the variational bicomplex to connect the geometry of the base manifold to the geometry of the fibre. This leads to a generalization of (1.1) and (1.2), and to a novel class of multisymplectic structures. Furthermore, it provides a completely coordinate-free characterization of multisymplectic systems.

We begin by outlining some of the key features of the variational bicomplex, which was introduced independently by Vinogradov [25; 26] and TulczYJEw [24]; we follow Anderson's presentation in $[3 ; 4]$. The variational bicomplex is constructed by splitting the exterior derivative into horizontal (base space) and vertical (fibre) components. In $\S 3$, we examine the implications of this splitting for Hamiltonian ODEs, leading to a coordinate-free approach that has a natural generalization to PDEs. With this approach, which is described in $\S 4$, a multisymplectic system on a Riemannian manifold is defined to be a set of first-order PDEs of the 
following form (in local coordinates):

$$
\left(\frac{\partial L_{\beta}^{i}}{\partial u^{\alpha}}-\frac{\partial L_{\alpha}^{i}}{\partial u^{\beta}}\right) u_{, i}^{\beta}-\frac{1}{\sqrt{g}} \frac{\partial}{\partial x^{i}}\left(\sqrt{g} L_{\alpha}^{i}\right)-\frac{\partial H}{\partial u^{\alpha}}=0 .
$$

Here $L_{\alpha}^{i}$ and $H$ are functions of the independent variables $x^{j}$ and the dependent variables $u^{\beta}$. The reason for this definition is that it is an expression in coordinates of something more fundamental, namely "conservation of symplecticity." For Hamiltonian ODEs, the (closed) symplectic 2-form is conserved by the flow. In the same way, for any system of the form (1.4), there is a closed 2-form associated with each independent variable, and these 2-forms are the components of a conservation law. By starting with this collection of 2 -forms, we are able to define multimomentum maps that yield further conservation laws and also to develop the idea of multisymplectic relative equilibria.

For Riemannian manifolds, the total exterior algebra (TEA) bundle of BRIDGES [8] generalizes the cotangent bundle for ODEs. Thus the TEA bundle is a rich source of multisymplectic PDEs, as described in $\S 5$. We find that every one-parameter Lie group of point transformations that preserve the multisymplectic structure is constrained by $(n-1) 2^{n}$ relations between first-order derivatives of the corresponding multimomentum map.

\section{The variational bicomplex}

This section outlines the key features of the variational bicomplex, following ANDERSON [3; 4]. Given a differential equation with independent variables $x=\left(x^{1}, \ldots, x^{n}\right) \in X$ and dependent variables $u=\left(u^{1}, \ldots, u^{m}\right) \in U$, each solution $u=f(x)$ can be represented as a section $s(x)=(x, f(x))$ in the trivial bundle $\pi: X \times U \rightarrow X$ with $\pi(x, u)=x$. [Note: Coordinates $(x, u)$ need only be local coordinates on $X \times U$.] However, to represent the differential equation itself (or its symmetries, conservation laws, Lagrangians, etc.), the proper setting is the infinite jet bundle $J^{\infty}(X \times U)$. This has local coordinates $\left(x, u, u_{\{1\}}, u_{\{2\}}, \ldots\right)$, where $u_{\{k\}}$ denotes the set of all $k^{\text {th }}$-order derivatives of $u$. Specifically,

$$
u_{\{1\}}=\left\{u_{i}^{\alpha}\right\}, \quad u_{\{2\}}=\left\{u_{i j}^{\alpha}\right\}, \quad \text { etc. }
$$

where any section $s(x)=(x, f(x))$ of $X \times U$ is prolonged to $J^{\infty}(X \times U)$ as follows:

$$
u_{i}^{\alpha}=\frac{\partial f^{\alpha}(x)}{\partial x^{i}}, \quad u_{i j}^{\alpha}=\frac{\partial^{2} f^{\alpha}(x)}{\partial x^{i} \partial x^{j}}, \quad \text { etc. }
$$

The trivial bundle $\pi^{\infty}: J^{\infty}(X \times U) \rightarrow X$ has projections $\pi^{\infty}\left(x, u, u_{\{1\}}, \ldots\right)=x$, and the fibres $\left(\pi^{\infty}\right)^{-1}(x)$ are equivalence classes of prolonged local sections. [Two sections are equivalent at $x$ if all of their derivatives agree there.] Given a section $s$, the equivalence class of its prolongation to all orders is denoted by $j^{\infty}(s)$.

Now consider the set $\Omega$ of differential forms on $J^{\infty}(X \times U)$. A contact form is a differential form $\theta \in \Omega$ such that

$$
\left[j^{\infty}(s)\right]^{*}(\theta)=0
$$


for every local section $s$ of $X \times U$. Crucially, the set of contact forms is a differential ideal in $\Omega$, so it induces a natural splitting of $\Omega$. A basis for this ideal is the set of differential one-forms

$$
\theta^{\alpha}=\mathrm{d} u^{\alpha}-u_{j}^{\alpha} \mathrm{d} x^{j}, \quad \theta_{i}^{\alpha}=\mathrm{d} u_{i}^{\alpha}-u_{i j}^{\alpha} \mathrm{d} x^{j}, \quad \text { etc. }
$$

(The Einstein summation convention is used throughout this paper.) So a basis for the set of all one-forms is

$$
\mathrm{d} x^{i}, \theta^{\alpha}, \theta_{i}^{\alpha}, \theta_{i j}^{\alpha}, \ldots,
$$

and this basis extends to a basis for $\Omega$ via the wedge product. A differential $(k+l)$-form $\mu$ is said to be of type $(k, l)$ if it can be written in local coordinates as a sum of terms of the form

$$
\mu=f[u] \mathrm{d} x^{i_{1}} \wedge \cdots \wedge \mathrm{d} x^{i_{k}} \wedge \theta_{\mathbf{J}_{1}}^{\alpha_{1}} \wedge \cdots \wedge \theta_{\mathbf{J}_{l}}^{\alpha_{l}}
$$

Here $f[u]$ is a smooth function of $x, u, u_{\{1\}}, \ldots, u_{\{K\}}$, for some $K \in \mathbb{N}_{0}$; if a multi-index $\mathbf{J}_{j}$ is null then $\theta_{\mathbf{J}_{j}}^{\alpha_{j}}$ is replaced by $\theta^{\alpha_{j}}$.

The exterior derivative of a function $f[u]$ may be written as the sum of a $(1,0)$-form and a (0,1)-form, as follows:

$$
\mathrm{d}(f[u])=D_{i}(f[u]) \mathrm{d} x^{i}+\frac{\partial f[u]}{\partial u_{\mathbf{J}}^{\alpha}} \theta_{\mathbf{J}}^{\alpha} .
$$

Here $D_{i}$ represents the total derivative with respect to $x^{i}$ :

$$
D_{i}=\frac{\partial}{\partial x^{i}}+u_{i}^{\alpha} \frac{\partial}{\partial u^{\alpha}}+u_{i j}^{\alpha} \frac{\partial}{\partial u_{j}^{\alpha}}+\cdots .
$$

This motivates the splitting of $d$ into horizontal and vertical derivatives:

$$
\mathrm{d}=\mathrm{d}_{\mathrm{h}}+\mathrm{d}_{\mathrm{v}}
$$

where

$$
\mathrm{d}_{\mathrm{h}}=\mathrm{d} x^{i} \wedge D_{i}, \quad \mathrm{~d}_{\mathrm{v}}=\theta_{\mathbf{J}}^{\alpha} \wedge \frac{\partial}{\partial u_{\mathbf{J}}^{\alpha}} .
$$

Simple calculations, together with the identity $\mathrm{d}^{2}=0$, yield

$$
\mathrm{d}_{\mathrm{h}}^{2}=0, \quad \mathrm{~d}_{\mathrm{h}} \mathrm{d}_{\mathrm{v}}=-\mathrm{d}_{\mathrm{v}} \mathrm{d}_{\mathrm{h}}, \quad \mathrm{d}_{\mathrm{v}}^{2}=0 .
$$

These results give the identities

$$
\mathrm{d}_{\mathrm{h}}\left(\mathrm{d} x^{i}\right)=0, \quad \mathrm{~d}_{\mathrm{v}}\left(\mathrm{d} x^{i}\right)=0, \quad \mathrm{~d}_{\mathrm{h}}\left(\theta_{\mathbf{J}}^{\alpha}\right)=\mathrm{d} x^{i} \wedge \theta_{\mathbf{J} i}^{\alpha}, \quad \mathrm{d}_{\mathbf{v}}\left(\theta_{\mathbf{J}}^{\alpha}\right)=0 .
$$

Let $\Omega^{k, l}$ denote the set of all differential $(k, l)$-forms over $J^{\infty}(X \times U)$. Then, from (2.1), the operations

$$
\mathrm{d}_{\mathrm{h}}: \Omega^{k, l} \rightarrow \Omega^{k+1, l}, \quad \mathrm{~d}_{\mathrm{v}}: \Omega^{k, l} \rightarrow \Omega^{k, l+1},
$$

yield a double complex, which is called the variational bicomplex. A differential form $\omega \in \Omega^{k, l}$ is horizontally closed if $\mathrm{d}_{\mathrm{h}} \omega=0$, and is horizontally exact (for $k \geq 1$ ) if there exists $\eta \in \Omega^{k-1, l}$ such that $\omega=d_{h} \eta$. Vertically closed and exact forms are defined similarly, with $d_{v}$ replacing $d_{h}$. A schematic of the variational bicomplex is shown below. 


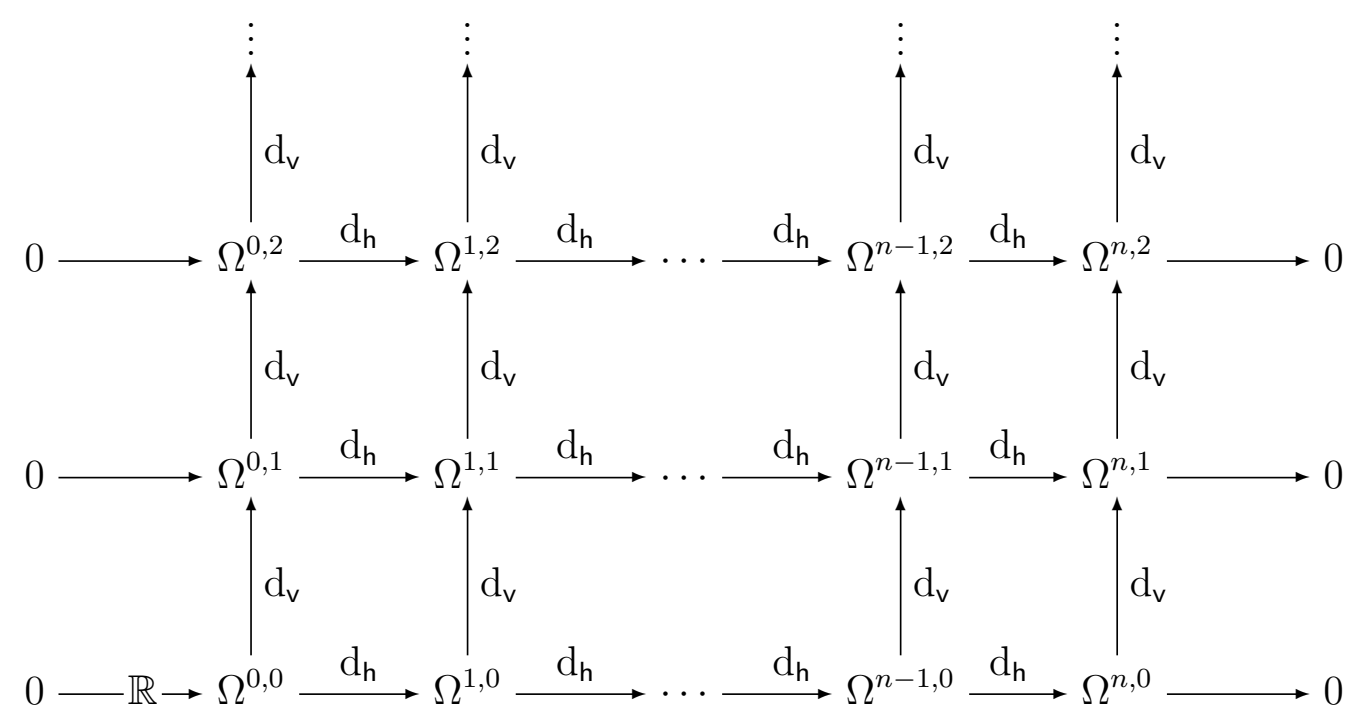

Each column is analogous to the de Rham complex for an infinite-dimensional space; for $l \geq 1$, every vertically closed $(k, l)$-form is vertically exact. At first sight, the horizontal rows of the variational bicomplex also seem to be analogous to the de Rham complex. However, the analogue of the Poincaré Lemma fails, because every $(n, l)$-form is horizontally closed, but most of these forms are not locally horizontally exact. Nevertheless, for $l \geq 1$, the rows can be made globally exact by augmenting the right-hand side of the complex, as shown below.

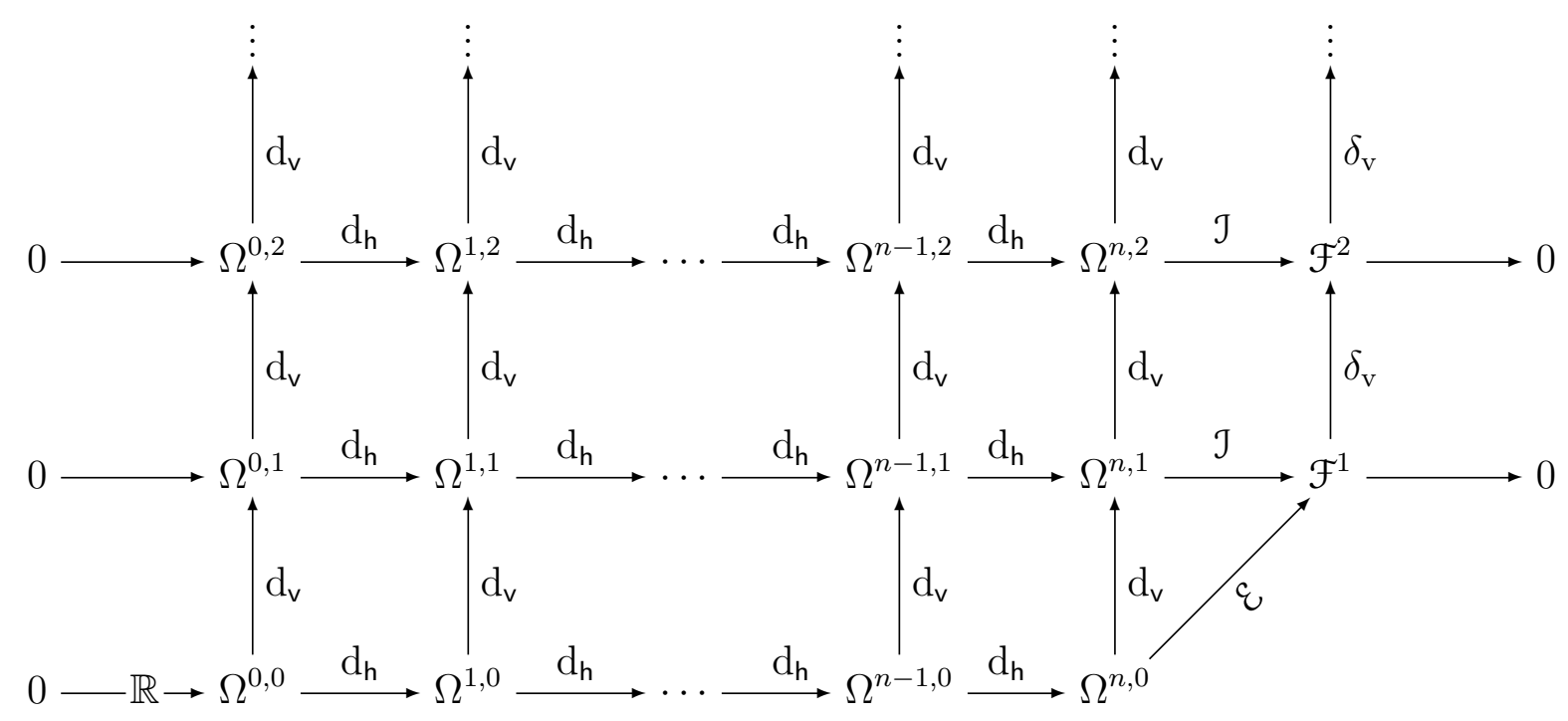

Here $\mathcal{J}: \Omega^{n, l} \rightarrow \Omega^{n, l}$ is the interior Euler operator, which is defined by

$$
\left.\left.\left.\mathcal{J}(\omega)=\frac{1}{l} \theta^{\alpha} \wedge\left[\left(\frac{\partial}{\partial u^{\alpha}}\right\rfloor \omega\right)-D_{i}\left(\frac{\partial}{\partial u_{i}^{\alpha}}\right\lrcorner \omega\right)+D_{i} D_{j}\left(\frac{\partial}{\partial u_{i j}^{\alpha}}\right\rfloor \omega\right)-\cdots\right],
$$


and $\mathcal{F}^{l}=\mathfrak{J}\left(\Omega^{n, l}\right)$. Furthermore, $\mathcal{E}=\mathcal{J}_{\mathrm{v}}$ is the Euler-Lagrange operator: given a Lagrangian $\mathscr{L} \in \Omega^{n, 0}$, the corresponding Euler-Lagrange equation amounts to $\mathcal{E}(\mathscr{L})=0$. (Bearing in mind that $\mathcal{F}^{l} \subset \Omega^{n, l}$, the operators $\delta_{\mathrm{v}}=\mathcal{J}_{\mathrm{v}}$ applied to elements of $\mathcal{F}^{l}$ correspond to higher variations.) The following properties of the interior Euler operator are particularly useful:

(i) $\mathcal{J}\left(\mathrm{d}_{\mathrm{h}} \mu\right)=0, \quad \forall \mu \in \Omega^{n-1, l}, \quad l \geq 1$;

(ii) For each $\omega \in \Omega^{n, l}, l \geq 1$, there exists $\mu \in \Omega^{n-1, l}$ such that $\omega=\mathfrak{J}(\omega)+\mathrm{d}_{\mathrm{h}}(\mu)$. Combining this with (i) yields $\mathfrak{J}^{2}=\mathfrak{J}$.

Although we have written $d_{h}$ and $d_{v}$ in terms of local coordinates, the above construction is coordinate-independent. Furthermore, it can be extended to any fibre bundle $\pi: E \rightarrow X$, not just trivial bundles. Crucially, for $l \geq 1$, each row of the augmented variational bicomplex is globally exact.

We have not imposed any constraints on the variational bicomplex because we seek general results about the structure of multisymplectic systems. However, it is important to be aware that when the bicomplex is pulled back to a given (prolonged) system of differential equations, the local cohomology typically ceases to be trivial (in columns containing $\Omega^{n-1, l}$ and $\Omega^{n, l}$ ) even when $X$ and $U$ have trivial de Rham cohomology. In particular, the nontrivial cohomology classes yield conservation laws and information about the existence of a Lagrangian, Darboux integrability, etc. As we are not using the pulled-back bicomplex, we shall be careful to identify which of our results apply only "on solutions" of a multisymplectic system.

Consider a Lie group $G$ that acts vertically on $J^{\infty}(E)$; we denote the associated Lie algebra by $\mathfrak{g}$. Each $\xi \in \mathfrak{g}$ has a corresponding infinitesimal generator, namely the vertical vector field

$$
\mathbf{v}_{\xi}=\left.\left(\frac{\mathrm{d}}{\mathrm{d} s} \exp (s \xi)(x, u)\right)\right|_{s=0} .
$$

Note that although $\mathbf{v}_{\xi}$ has no horizontal $(x)$ components, its coefficients may depend on $x$. Let $\left.Q^{\alpha}=\mathbf{v}_{\xi}\right\lrcorner \theta^{\alpha}$; then the infinitesimal generator is prolonged to $J^{\infty}(E)$ as follows:

$$
\mathbf{v}_{\xi}=Q^{\alpha} \frac{\partial}{\partial u^{\alpha}}+\left(D_{i} Q^{\alpha}\right) \frac{\partial}{\partial u_{i}^{\alpha}}+\left(D_{i} D_{j} Q^{\alpha}\right) \frac{\partial}{\partial u_{i j}^{\alpha}}+\cdots .
$$

[From here on, $\mathbf{v}_{\xi}$ denotes the prolonged generator.] The following useful identities hold for all $\omega \in \Omega^{k, l}$ :

$$
\begin{gathered}
\left.\left.\mathbf{v}_{\xi}\right\lrcorner \mathrm{d}_{\mathbf{h}}(\omega)=-\mathrm{d}_{\mathbf{h}}\left(\mathbf{v}_{\xi}\right\lrcorner \omega\right) ; \\
\left.\left.\mathcal{L}_{\mathbf{v}_{\xi}}(\omega)=\mathrm{d}_{\mathbf{v}}\left(\mathbf{v}_{\xi}\right\lrcorner \omega\right)+\mathbf{v}_{\xi}\right\lrcorner \mathrm{d}_{\mathbf{v}} \omega .
\end{gathered}
$$

The second of these uses the first to replace $d$ by $d_{v}$ in Cartan's formula for the Lie derivative.

Noether's Theorem has a very elegant formulation in terms of vertical and horizontal derivatives. Given $\mathscr{L} \in \Omega^{n, 0}$, the identity (ii) above yields

$$
\mathrm{d}_{\mathrm{v}} \mathscr{L}=\mathcal{E}(\mathscr{L})-\mathrm{d}_{\mathrm{h}} \eta
$$

for some $\eta \in \Omega^{n-1,1}$. The vector field $\mathbf{v}_{\xi}$ generates variational symmetries if there exists $\sigma_{\xi} \in \Omega^{n-1,0}$ such that

$$
\left.\mathbf{v}_{\xi}\right\lrcorner \mathrm{d}_{\mathrm{v}} \mathscr{L}=\mathrm{d}_{\mathrm{h}} \sigma_{\xi} .
$$


Then (2.2) implies that

$$
\left.\left.\mathrm{d}_{\mathrm{h}}\left(\sigma_{\xi}-\mathbf{v}_{\xi}\right\lrcorner \eta\right)=\mathbf{v}_{\xi}\right\lrcorner \mathcal{E}(\mathscr{L})
$$

which leads to the conservation law

$$
\left.\mathrm{d}_{\mathrm{h}}\left(\sigma_{\xi}-\mathbf{v}_{\xi}\right\lrcorner \eta\right)=0 \quad \text { on solutions of } \quad \mathcal{E}(\mathscr{L})=0 .
$$

This is Noether's Theorem in its most general setting.

\section{Classical mechanics via the variational bicomplex}

A standard approach to classical mechanics begins with a closed nondegenerate two-form $\omega$ on a manifold $U$; one then studies paths in this manifold. In this section it is shown how this view can be re-interpreted by including the geometry of time and using the variational bicomplex. Attention will be restricted to the simplest case, where $X=\mathbb{R}, U=\mathbb{R}^{2}$, and canonical coordinates $(t, q, p)$ are used for $E=X \times U$.

The standard approach for $\omega=\mathrm{d} p \wedge \mathrm{d} q$ is as follows. Let $\mathbf{v}: U \rightarrow T U$ be a generator of a one-parameter Lie group of diffeomorphisms that preserves $\omega$, so that

$$
\left.\left.\left.0=\mathcal{L}_{\mathbf{v}} \omega=\mathbf{v}\right\rfloor \mathrm{d} \omega+\mathrm{d}(\mathbf{v}\rfloor \omega\right)=\mathrm{d}(\mathbf{v}\rfloor \omega\right) .
$$

As $X \times U$ is topologically trivial, there exists a function $H: U \rightarrow \mathbb{R}$ such that

$$
\mathbf{v}\rfloor \omega=-\mathrm{d} H .
$$

This implies that, in terms of the coordinates,

$$
\mathbf{v}=\frac{\partial H}{\partial p} \frac{\partial}{\partial q}-\frac{\partial H}{\partial q} \frac{\partial}{\partial p} .
$$

The flow is given by

$$
\left.\left.D_{t}(q)=\mathbf{v}\right\rfloor \mathrm{d} q=\frac{\partial H}{\partial p}, \quad D_{t}(p)=\mathbf{v}\right\rfloor \mathrm{d} p=-\frac{\partial H}{\partial q} .
$$

This approach implicitly restricts attention to autonomous Hamiltonian systems, although nonautonomous Hamiltonian systems can be treated by increasing the dimension of $U$, introducing an extra pair of canonical coordinates $q_{2}=H$ and $p_{2}=t$.

Now reconsider this problem from the viewpoint of the variational bicomplex. In this setting the "autonomization trick" becomes superfluous. Let $\omega$ be the $(0,2)$-form:

$$
\omega=\mathrm{d}_{\mathrm{v}} p \wedge \mathrm{d}_{\mathrm{v}} q .
$$

Then, if a vertical vector field,

$$
\mathbf{v}_{\xi}=v_{q} \frac{\partial}{\partial q}+v_{p} \frac{\partial}{\partial p}
$$

generates a one-parameter group of $\omega$-preserving diffeomorphisms, a similar argument to the above leads to

$$
\left.\mathbf{v}_{\xi}\right\lrcorner \omega=-\mathrm{d}_{\mathbf{v}} H+F(t) \mathrm{d} t,
$$


for some functions $H$ and $F$. As $\omega$ is a $(0,2)$-form, it follows that $F(t)=0$, and so

$$
v_{q}=\frac{\partial H}{\partial p} \quad \text { and } \quad v_{p}=-\frac{\partial H}{\partial q}
$$

note that $H$ may depend explicitly on $t$. It is a simple exercise ${ }^{1}$ to show that, on solutions of $(3.1)$,

$$
\mathrm{d}_{\mathrm{v}} p \wedge \mathrm{d}_{\mathrm{v}} q=\mathrm{d} p \wedge \mathrm{d} q+\mathrm{d} p_{2} \wedge \mathrm{d} q_{2} .
$$

This identity holds whether or not the system is autonomous; the advantage of using (3.2) instead of the standard symplectic two-form is that horizontal and vertical components are distinguished.

Furthermore, (3.2) enables us to write down the flow in a completely coordinate-free way as follows:

$$
\mathrm{d}_{\mathrm{h}} \omega=0 \quad \text { on solutions of }(3.1) .
$$

To see this, expand the left-hand side of (3.3) to obtain

$$
\begin{aligned}
\mathrm{d}_{\mathrm{h}} \omega & =\left(\mathrm{d}_{\mathrm{h}} \mathrm{d}_{\mathrm{v}} p\right) \wedge \mathrm{d}_{\mathrm{v}} q-\mathrm{d}_{\mathrm{v}} p \wedge\left(\mathrm{d}_{\mathrm{h}} \mathrm{d}_{\mathrm{v}} q\right) \\
& =-\mathrm{d}_{\mathrm{v}}\left(\mathrm{d}_{\mathrm{h}} p \wedge \mathrm{d}_{\mathrm{v}} q+\mathrm{d}_{\mathrm{v}} p \wedge \mathrm{d}_{\mathrm{h}} q\right)
\end{aligned}
$$

So there exists a $(1,0)$-form $S$ such that, on solutions,

$$
\mathrm{d}_{\mathrm{h}} p \wedge \mathrm{d}_{\mathrm{v}} q+\mathrm{d}_{\mathrm{v}} p \wedge \mathrm{d}_{\mathrm{h}} q=\mathrm{d}_{\mathrm{v}} S .
$$

In coordinates, write $S=H \mathrm{~d} t$. Then (3.4) amounts to

$$
D_{t}(q) \mathrm{d}_{\mathrm{v}} p \wedge \mathrm{d} t-D_{t}(p) \mathrm{d}_{\mathrm{v}} q \wedge \mathrm{d} t=\mathrm{d}_{\mathrm{v}} H \wedge \mathrm{d} t .
$$

Consequently, $H$ is a function of $(t, q, p)$ only and

$$
D_{t}(q)=\frac{\partial H}{\partial p} \quad \text { and } \quad D_{t}(p)=-\frac{\partial H}{\partial q} .
$$

Conversely, if (3.5) holds then the above argument in reverse implies that $\mathrm{d}_{\mathrm{h}} \omega=0$ on all solutions of (3.5).

The above construction of classical mechanics in terms of horizontal and vertical derivatives is genuinely coordinate-free; the base space coordinate $t$ appears only when $d_{h}$ is evaluated in terms of coordinates. Moreover, there is no need to restrict attention to $X=\mathbb{R}$.

Theorem 3.1 Let $X$ be a one-dimensional Riemannian manifold, and suppose that $E=X \times U$ is equipped with a vertically closed, nondegenerate $(0,2)$-form $\omega$. Then there exists a locally Hamiltonian flow if and only if $\mathrm{d}_{\mathrm{h}} \omega=0$ on all solutions of the equations of motion.

The nondegeneracy condition implies that $U$ is even-dimensional, but it does not otherwise constrain the global structure of $U$. So given a vertically closed nondegenerate $(0,2)-$ form $\omega$, the existence of locally Hamiltonian dynamics is determined by the condition (3.3). We now generalize this idea to include multisymplectic systems of PDEs, with the added freedom that (multi-)symplectic structures are no longer required to be nondegenerate.

\footnotetext{
${ }^{1}$ We are grateful to Gianne Derks for this observation.
} 


\section{Multisymplectic PDEs and the variational bicomplex}

The key feature of multisymplectic systems is the existence of a vertically closed two-form $\kappa^{i}$ associated with each independent variable $x^{i}$; specifically,

$$
\kappa^{i}=\sum_{\alpha<\beta} f_{\alpha \beta}^{i}(x, u) \mathrm{d}_{\mathrm{v}} u^{\alpha} \wedge \mathrm{d}_{\mathrm{v}} u^{\beta}
$$

for some smooth functions $f_{\alpha \beta}^{i}$. These two-forms satisfy a conservation law that, if $x^{i}$ are cartesian coordinates on $\mathbb{R}^{n}$, amounts to

$$
D_{i}\left(\kappa^{i}\right)=0,
$$

on all solutions of the given system of PDEs. To extend this to a general Riemannian manifold, $X$, let vol denote the volume form on $X$; in coordinates, this is the $(n, 0)$-form

$$
\mathrm{vol}=\sqrt{g} \mathrm{~d} x^{1} \wedge \cdots \wedge \mathrm{d} x^{n},
$$

where $g$ is the determinant of the metric tensor $g_{i j}$. Let

$$
\boldsymbol{\kappa}=\kappa^{i} D_{i}
$$

be a vector whose components, $\kappa^{i}$, are vertically closed $(0,2)$-forms. Define the vertically closed $(n-1,2)$-form

$$
\omega=\kappa\lrcorner \operatorname{vol} .
$$

Then conservation of symplecticity corresponds to the condition $d_{h} \omega=0$. To see this, work in coordinates to obtain

$$
\left.\mathrm{d}_{\mathrm{h}} \omega=\mathrm{d}_{\mathrm{h}}\left(\sqrt{g} \kappa^{i}\right) \wedge\left(D_{i}\right\lrcorner\left(\mathrm{d} x^{1} \wedge \cdots \wedge \mathrm{d} x^{n}\right)\right)=\frac{1}{\sqrt{g}} D_{i}\left(\sqrt{g} \kappa^{i}\right) \wedge \text { vol }=(\text { Div } \boldsymbol{\kappa}) \wedge \text { vol } .
$$

The conservation law (4.1) arises in the special case when $X=\mathbb{R}^{n}$.

Conversely, suppose that $E=X \times U$ is equipped with a vertically closed $(n-1,2)$-form $\omega$ and that a quasilinear system of first-order PDEs satisfies the condition

$$
\mathrm{d}_{\mathrm{h}} \omega=0 \quad \text { (on all solutions). }
$$

Then it is easy to extract the components $\kappa^{i}$ that satisfy (4.3), and so the system is multisymplectic.

It is instructive to examine the consequences of (4.4) in detail. We shall work locally, applying the Poincaré Lemma to vertically closed forms. As $\omega$ is vertically closed, there exists $\eta \in \Omega^{n-1,1}$ such that

$$
\omega=\mathrm{d}_{\mathrm{v}} \eta
$$

Thus, on all solutions of the system,

$$
\mathrm{d}_{\mathrm{v}} \mathrm{d}_{\mathrm{h}} \eta=-\mathrm{d}_{\mathrm{h}} \mathrm{d}_{\mathrm{v}} \eta=-\mathrm{d}_{\mathrm{h}} \omega=0 .
$$

So (locally) there exists $\mathscr{L} \in \Omega^{n, 0}$ such that

$$
\mathrm{d}_{\mathrm{h}} \eta+\mathrm{d}_{\mathrm{v}} \mathscr{L}=0 \quad \text { (on all solutions). }
$$


In fact, $\mathscr{L}$ is a Lagrangian $(n, 0)$-form for the system of PDEs. To see this, apply the interior Euler operator $\mathcal{J}$ to $(4.6)$, which gives

$$
\mathcal{E}(\mathscr{L})=\mathcal{J ~ d}_{\mathrm{v}} \mathscr{L}=-\mathcal{J}_{\mathrm{h}} \eta \equiv 0 \quad \text { (on solutions) }
$$

This is the Euler-Lagrange equation for the system. HYDON [14] has shown how to proceed from a multisymplectic structure to an equivalent degenerate first-order Lagrangian structure (provided that the relevant cohomology classes are trivial). The above construction is a coordinate-free generalization of the process. Equation (4.6) is a quasi-conservation law in Hydon's terminology.

\subsection{Multimomentum maps}

For Hamiltonian systems, Noether's Theorem can be expressed in terms of a momentum map [19]. In this section the concept of a momentum map is combined with the variational bicomplex and applied to multisymplectic systems. Given a Lie group $G$ of $\omega$-preserving transformations, each vector field $\mathbf{v}_{\xi}$ with $\xi \in \mathfrak{g}$ satisfies the identity

$$
\left.0=\mathcal{L}_{\mathbf{v}_{\xi}}(\omega)=\mathrm{d}_{\mathbf{v}}\left(\mathbf{v}_{\xi}\right\lrcorner \omega\right)
$$

Now suppose that $\left.\mathbf{v}_{\xi}\right\lrcorner \omega$ is vertically exact, so that

$$
\left.\mathbf{v}_{\xi}\right\lrcorner \omega=\mathrm{d}_{\mathrm{v}} \lambda_{\xi}
$$

for some $\lambda_{\xi} \in \Omega^{n-1,0}$. Then a multimomentum map $J: E \rightarrow \mathfrak{g}^{*} \otimes \Omega^{n-1,0}$ is defined by

$$
J(x, u)(\xi)=\lambda_{\xi}(x, u) .
$$

Here $\mathfrak{g}^{*}$ is the dual of the Lie algebra. This property is to be compared with the multimomentum map in field theory, where the momentum map lies in $\mathfrak{g}^{*} \otimes \Omega^{n-1}(Z)$ with $Z$ the total bundle which models the phase space (see LAWSON [17] and references therein).

We now examine the additional conditions that are required for $d_{h} \lambda_{\xi}=0$ to be the conservation law (2.5) of the Euler-Lagrange equations that are associated with the multisymplectic system. Up to now, we have only sketched the link between the Lagrangian and multisymplectic formulations. Given a Lagrangian of the form

$$
\left.\mathscr{L}=L_{\beta}^{i}(x, u) \mathrm{d}_{\mathrm{h}} u^{\beta} \wedge\left(D_{i}\right\lrcorner \operatorname{vol}\right)-H(x, u) \operatorname{vol}
$$

the Euler-Lagrange equations are

$$
\left(\frac{\partial L_{\beta}^{i}}{\partial u^{\alpha}}-\frac{\partial L_{\alpha}^{i}}{\partial u^{\beta}}\right) D_{i} u^{\beta}-\frac{1}{\sqrt{g}} \frac{\partial}{\partial x^{i}}\left(\sqrt{g} L_{\alpha}^{i}\right)-\frac{\partial H}{\partial u^{\alpha}}=0 .
$$

It is easy to verify that $(2.2)$ yields the $(n-1,1)$-form

$$
\left.\eta=L_{\beta}^{i}(x, u) \mathrm{d}_{\mathrm{v}} u^{\beta} \wedge\left(D_{i}\right\rfloor \mathrm{vol}\right),
$$


which leads to the multisymplectic $(n-1,2)$-form

$$
\left.\omega=\frac{\partial L_{\beta}^{i}}{\partial u^{\alpha}} \mathrm{d}_{\mathrm{v}} u^{\alpha} \wedge \mathrm{d}_{\mathrm{v}} u^{\beta} \wedge\left(D_{i}\right\rfloor \operatorname{vol}\right) .
$$

Conversely, if $\omega$ is a vertically closed $(n-1,2)$-form such that $\mathrm{d}_{\mathrm{h}} \omega=0$ on solutions of a quasilinear system of first-order PDEs, there exists a corresponding Lagrangian of the form (4.10), provided that the relevant cohomology classes are trivial. So Noether's Theorem (2.5) for the Lagrangian formulation should lead to a multimomentum map (4.9), namely

$$
\left.\lambda_{\xi}=\sigma_{\xi}-\mathbf{v}_{\xi}\right\lrcorner \eta
$$

It is useful to write $\lambda_{\xi}$ as follows:

$$
\left.\lambda_{\xi}=\lambda_{\xi}^{i}(x, u) D_{i}\right\rfloor \mathrm{vol}
$$

Then the multisymplectic and Lagrangian formulations together yield the conditions

$$
\begin{aligned}
\mathrm{d}_{\mathrm{v}} \lambda_{\xi} & \left.=\mathbf{v}_{\xi}\right\lrcorner \omega \\
\mathrm{d}_{\mathrm{h}} \lambda_{\xi} & \left.=\mathbf{v}_{\xi}\right\lrcorner \mathcal{E}(\mathscr{L}) .
\end{aligned}
$$

By using (4.16), it is straightforward to reduce (4.17) to

$$
\left.\frac{1}{\sqrt{g}} \frac{\partial}{\partial x^{i}}\left(\sqrt{g} \lambda_{\xi}^{i}\right)+\frac{Q^{\alpha}}{\sqrt{g}} \frac{\partial}{\partial x^{i}}\left(\sqrt{g} L_{\alpha}^{i}\right)+\mathbf{v}_{\xi}\right\lrcorner \mathrm{d}_{\mathbf{v}} H=0 .
$$

This is the extra constraint on $\lambda_{\xi}$ that yields Noether's Theorem.

Theorem 4.1 Any multimomentum map $J(x, u)(\xi)=\lambda_{\xi}$ that satisfies (4.16) and (4.18) gives rise to the conservation law $\mathrm{d}_{\mathrm{h}} \lambda_{\xi}=0$ on solutions of the multisymplectic system (4.11).

\subsection{Multisymplectic relative equilibria}

One of the most useful tools in the application of multisymplectic structures to nonlinear PDEs is the concept of a multisymplectic relative equilibrium (MSRE) [9]. In particular, the geometry of a MRSE leads to a simple criterion for nonlinear waves to be unstable. The following definition of a MSRE generalizes the classical definition of a relative equilibrium of a Hamiltonian system of ODEs [2], and gives a coordinate-free generalization of the MSRE introduced in [6; 9]. Given a vertical symmetry generator $\mathbf{v}_{\xi}=Q^{\alpha} \partial_{u^{\alpha}}$, a MSRE is a solution of $\mathcal{E}(\mathscr{L})=0$ that is of the form

$$
u(x)=e^{s(x) \mathbf{v}_{\xi}} u_{0},
$$

where $s$ is a linear function of $x$. Let $u\left(x_{0}\right)=u_{0}$; then $s(x)=c_{i}\left(x^{i}-x_{0}^{i}\right)$. Note that

$$
u^{\alpha}(x)=u_{0}^{\alpha}+s(x) Q^{\alpha}\left(x_{0}, u_{0}\right)+O\left(\left|x-x_{0}\right|^{2}\right) .
$$

Thus, by expanding $\mathcal{E}(\mathscr{L})=0$ about $\left(x_{0}, u_{0}\right)$, we obtain the condition

$$
0=\left.\mathcal{E}(\mathscr{L})\right|_{\left(x_{0}, u_{0}\right)}=\left.\left\{\left(\left(\frac{\partial L_{\beta}^{i}}{\partial u^{\alpha}}-\frac{\partial L_{\alpha}^{i}}{\partial u^{\beta}}\right) c_{i} Q^{\alpha}-\frac{\partial H}{\partial u^{\beta}}\right) \theta^{\beta} \wedge \operatorname{vol}\right\}\right|_{\left(x_{0}, u_{0}\right)} .
$$


This can be simplified with the aid of (4.16), which amounts to

$$
Q^{\alpha}\left(\frac{\partial L_{\beta}^{i}}{\partial u^{\alpha}}-\frac{\partial L_{\alpha}^{i}}{\partial u^{\beta}}\right)=\frac{\partial \lambda_{\xi}^{i}}{\partial u^{\beta}} .
$$

So the condition for a MSRE is

$$
\left.\left\{c_{i} \frac{\partial \lambda_{\xi}^{i}}{\partial u^{\beta}}-\frac{\partial H}{\partial u^{\beta}}\right\}\right|_{\left(x_{0}, u_{0}\right)}=0 .
$$

In other words, $u_{0}$ is an extremum of $H$ (treating $x_{0}$ as a parameter) subject to the constraint that $\lambda_{\xi}$ is fixed. This is analogous to the situation for the relative equilibria of Hamiltonian ODEs. The condition (4.19) may also be written in coordinate-free notation, as follows:

$$
0=\left.\mathcal{E}(\mathscr{L})\right|_{\left(x_{0}, u_{0}\right)}=-\left.\left(\mathrm{d}_{\mathrm{h}} s \wedge \mathrm{d}_{\mathrm{v}} \lambda_{\xi}+\mathrm{d}_{\mathrm{v}} H \wedge \operatorname{vol}\right)\right|_{\left(x_{0}, u_{0}\right)} .
$$

Note that $\mathrm{d}_{\mathrm{h}} s$ is constant. Applications of MSRE can be found in $[6 ; 7 ; 9]$.

\section{Multisymplectic systems on the TEA bundle}

From here on, we shall restrict attention to a particularly important class of systems, namely those that are built on the total exterior algebra (TEA) bundle. This is the space whose sections are

$$
\widetilde{u}=\left(u^{(0)}, u^{(1)}, \ldots, u^{(n)}\right),
$$

where each $u^{(k)}$ is a mapping from $X$ into $\Omega^{k, 0}$. In terms of the notation of $\S 2$, the space $U$ is coordinatized by $u=\left(u^{1}, \ldots, u^{m}\right)$, where $u^{i}$ are the $m=2^{n}$ coefficients of the differential forms in $\widetilde{u}$.

The TEA bundle uses a horizontal Hodge dual operator, $\star: \Omega^{k, l} \rightarrow \Omega^{n-k, l}$, which, when $l=0$, is defined exactly as the usual Hodge star; it is normalized by

$$
g_{x}^{*}(\mathbf{a}, \mathbf{b}) \operatorname{vol}=\mathbf{a} \wedge \star \mathbf{b}, \quad \mathbf{a}, \mathbf{b} \in \Omega^{k, 0}(X) .
$$

In particular, the following identities hold for any $\omega, \eta \in \Omega^{k, 0}$ :

$$
\omega \wedge \star \eta=\eta \wedge \star \omega, \quad \omega \wedge \star \mathrm{d}_{\mathrm{v}} \eta=(-1)^{k} \mathrm{~d}_{\mathrm{v}} \eta \wedge \star \omega .
$$

The action of $\star$ on vertical components is trivial:

$$
\mathrm{d}_{\mathrm{v}} \star \omega=\star \mathrm{d}_{\mathrm{v}} \omega, \quad \text { for any } \omega \in \Omega^{k, l} \text {. }
$$

Thus the action on $(k, l)$-forms is obtained by pulling $\star$ through any vertical components, leaving it to act solely on the horizontal part of the form.

Later, we will use the horizontal codifferential $\delta_{\mathrm{h}}: \Omega^{k, l} \rightarrow \Omega^{k-1, l}$, which is defined by

$$
\delta_{\mathrm{h}} \omega=(-1)^{n k+k+1} \star \mathrm{d}_{\mathrm{h}} \star \omega .
$$


By applying the standard identity $\star \star \omega=(-1)^{k(n-k)} \omega$ we obtain

$$
\mathrm{d}_{\mathrm{h}} \star \omega=(-1)^{k} \star \delta_{\mathrm{h}} \omega .
$$

BRIDGES [8] introduced the following natural $(n, 0)$-form on the TEA bundle:

$$
\Theta=\sum_{k=1}^{n} \mathrm{~d}_{\mathbf{h}} u^{(k-1)} \wedge \star u^{(k)} .
$$

In particular, when $n=1$ and $\widetilde{u}=(q, p \mathrm{~d} t)$, this form is

$$
\Theta=p \mathrm{~d}_{\mathrm{h}} q=p D_{t}(q) \mathrm{d} t .
$$

As $\Theta$ is linear in the first derivatives of $u$, we can examine the multisymplectic structure that arises by treating it as a Lagrangian. The case $n=1$ shows that this is a natural generalization of the Hamiltonian formulation. Following the construction in $\S 4$, set

$$
\eta=\sum_{k=1}^{n} \mathrm{~d}_{\mathrm{v}} u^{(k-1)} \wedge \star u^{(k)} .
$$

The interesting feature in going from (5.5) to (5.6) is the shift from horizontal exterior derivatives $\mathrm{d}_{\mathrm{h}}$ to vertical derivatives $\mathrm{d}_{\mathrm{v}}$. When $n=1$, this amounts to $\eta=p \mathrm{~d}_{\mathrm{v}} q$, which is the familiar canonical one-form on the cotangent bundle.

Taking the horizontal exterior derivative of $\eta$ gives

$$
\begin{aligned}
\mathrm{d}_{\mathrm{h}} \eta & =\sum_{k=1}^{n}\left(\mathrm{~d}_{\mathrm{h}} \mathrm{d}_{\mathfrak{v}} u^{(k-1)}\right) \wedge \star u^{(k)}+\sum_{k=1}^{n}(-1)^{k} \mathrm{~d}_{\mathrm{v}} u^{(k-1)} \wedge \mathrm{d}_{\mathrm{h}} \star u^{(k)} \\
& =-\mathrm{d}_{\mathbf{v}} \Theta+\sum_{k=1}^{n}(-1)^{k} \mathrm{~d}_{\mathrm{h}} u^{(k-1)} \wedge \star \mathrm{d}_{\mathrm{v}} u^{(k)}+\sum_{k=1}^{n} \mathrm{~d}_{\mathrm{v}} u^{(k-1)} \wedge \star \delta_{\mathrm{h}} u^{(k)},
\end{aligned}
$$

and so

$$
\mathrm{d}_{\mathrm{h}} \eta+\mathrm{d}_{\mathrm{v}} \Theta=\sum_{k=1}^{n}\left(\mathrm{~d}_{\mathrm{v}} u^{(k)} \wedge \star \mathrm{d}_{\mathrm{h}} u^{(k-1)}+\mathrm{d}_{\mathrm{v}} u^{(k-1)} \wedge \star \mathrm{d}_{\mathrm{h}} u^{(k)}\right) .
$$

The right-hand side of (5.7) is invariant under the internal Euler operator $\mathcal{J}$, so

$$
\mathcal{E}(\boldsymbol{\Theta})=\mathcal{J}_{\mathrm{v}} \boldsymbol{\Theta}=\sum_{k=1}^{n}\left(\mathrm{~d}_{\mathrm{v}} u^{(k)} \wedge \star \mathrm{d}_{\mathbf{h}} u^{(k-1)}+\mathrm{d}_{\mathrm{v}} u^{(k-1)} \wedge \star \mathrm{d}_{\mathbf{h}} u^{(k)}\right) .
$$

By equating coefficients of $\mathrm{d}_{\mathrm{v}} u^{(k)}$, the Euler-Lagrange equation $\mathcal{E}(\boldsymbol{\Theta})=0$ is decomposed into

$$
\left.\begin{array}{rl}
\delta_{\mathrm{h}} u^{(1)} & =0 ; \\
\mathrm{d}_{\mathrm{h}} u^{(k-1)}+\delta_{\mathrm{h}} u^{(k+1)} & =0, \quad k=1, \ldots, n-1 ; \\
\mathrm{d}_{\mathrm{h}} u^{(n-1)} & =0 .
\end{array}\right\}
$$

The left-hand side can be regarded as the multisymplectic Dirac operator $\mathbf{J}_{\boldsymbol{\partial}}[8]$, in the sense that $\mathbf{J}_{\boldsymbol{\partial}} \circ \mathbf{J}_{\boldsymbol{\partial}}=-\mathrm{Id} \otimes \Delta$ where $\Delta$ is the standard Laplacian on forms over $X$. Under suitable hypotheses, the kernel of $\mathbf{J}_{\boldsymbol{\partial}}$ consists of the harmonic forms on $X$. 
So (5.5) is associated with the multisymplectic structure

$$
\omega=\mathrm{d}_{\mathrm{v}} \eta=\sum_{k=1}^{n}(-1)^{k} \mathrm{~d}_{\mathrm{v}} u^{(k-1)} \wedge \star \mathrm{d}_{\mathrm{v}} u^{(k)}
$$

More generally, every Lagrangian of the form

$$
\mathscr{L}=\Theta-H(x, u) \operatorname{vol}
$$

is associated with the multisymplectic structure (5.10).

\subsection{Example: TEA bundle over a two-manifold}

As an illustration of the theory, take $X$ to be a smooth oriented Riemannian two-manifold. We need the identities

$$
\begin{aligned}
\star 1 & =\text { vol, } \quad \star \text { vol }=1, \\
\star \mathrm{d} x^{1} & =\frac{1}{\sqrt{g}}\left(g_{21} \mathrm{~d} x^{1}+g_{22} \mathrm{~d} x^{2}\right)=\sqrt{g}\left(g^{11} \mathrm{~d} x^{2}-g^{12} \mathrm{~d} x^{1}\right), \\
\star \mathrm{d} x^{2} & =-\frac{1}{\sqrt{g}}\left(g_{11} \mathrm{~d} x^{1}+g_{12} \mathrm{~d} x^{2}\right)=\sqrt{g}\left(g^{21} \mathrm{~d} x^{2}-g^{22} \mathrm{~d} x^{1}\right) .
\end{aligned}
$$

Now consider the form $\Theta$ on this manifold; in coordinates,

$$
\Theta=\mathrm{d}_{\mathrm{h}} q \wedge \star p+\mathrm{d}_{\mathrm{h}} p \wedge \star r
$$

where points in $\Omega(X)$ are represented by

$$
(q, p, r) \in \Omega^{0,0}(X) \times \Omega^{1,0}(X) \times \Omega^{2,0}(X) .
$$

Here

$$
p=p_{1} \mathrm{~d} x^{1}+p_{2} \mathrm{~d} x^{2}, \quad r=r_{12} \text { vol, }
$$

where $q, p_{1}, p_{2}$ and $r_{12}$ are smooth functions. (We use subscripts rather than superscripts as indices, to indicate the pairing of the coefficients with the basis that is a feature of the TEA bundle.) Substitution into (5.13) leads to

$$
\boldsymbol{\Theta}=\left[\frac{p_{1}}{g}\left(g_{22} D_{1}(q)-g_{12} D_{2}(q)\right)-\frac{p_{2}}{g}\left(g_{21} D_{1}(q)-g_{11} D_{2}(q)\right)+\frac{r_{12}}{\sqrt{g}}\left(D_{1}\left(p_{2}\right)-D_{2}\left(p_{1}\right)\right)\right] \text { vol } .
$$

By raising the indices on $p$, we obtain

$$
\Theta=\left[p^{1} D_{1}(q)+p^{2} D_{2}(q)+\frac{r_{12}}{\sqrt{g}}\left(D_{1}\left(p_{2}\right)-D_{2}\left(p_{1}\right)\right)\right] \text { vol } .
$$

This is the general local expression for $\Theta$ on a smooth oriented Riemannian manifold.

Taking $\Theta$ as the part of the Lagrangian that contains derivatives, and constructing the Euler-Lagrange equations gives the metric a more prominent role. For instance, suppose that

$$
H \mathrm{vol}=\frac{1}{2} p \wedge \star p+V(q) r
$$


where $V(q)$ is a given smooth function. Then the Lagrangian $\mathscr{L}=\Theta-H$ vol leads to the following multisymplectic PDE:

$$
\mathbf{J}_{1}(x) D_{1} u+\mathbf{J}_{2}(x) D_{2} u+\mathbf{K}(x) u=\left[V^{\prime}(q), g^{11} p_{1}+g^{12} p_{2}, g^{21} p_{1}+g^{22} p_{2}, V(q)\right]^{\mathrm{T}} .
$$

Here $u=\left[q, p_{1}, p_{2}, r_{12}\right]^{\mathrm{T}}$ and

$$
\mathbf{J}_{1}(x)=\left[\begin{array}{cccc}
0 & -g^{11} & -g^{21} & 0 \\
g^{11} & 0 & 0 & 0 \\
g^{21} & 0 & 0 & -g^{-1 / 2} \\
0 & 0 & g^{-1 / 2} & 0
\end{array}\right], \quad \mathbf{J}_{2}(x)=\left[\begin{array}{cccc}
0 & -g^{12} & -g^{22} & 0 \\
g^{12} & 0 & 0 & g^{-1 / 2} \\
g^{22} & 0 & 0 & 0 \\
0 & -g^{-1 / 2} & 0 & 0
\end{array}\right]
$$

and

$$
\mathbf{K}(x)=\left[\begin{array}{cccc}
0 & \sigma^{1} & \sigma^{1} & 0 \\
0 & 0 & 0 & 0 \\
0 & 0 & 0 & 0 \\
0 & 0 & 0 & 0
\end{array}\right]
$$

The coefficients $\sigma^{k}$ give curvature information, and can be expressed in terms of Christoffel symbols of the second kind by

$$
\sigma^{k}=-\frac{1}{\sqrt{g}} \frac{\partial}{\partial x^{l}}\left(\sqrt{g} g^{k l}\right)=g^{11} \Gamma_{11}^{k}+2 g^{12} \Gamma_{12}^{k}+g^{22} \Gamma_{22}^{k}, \quad k=1,2 .
$$

(Note: The Gaussian curvature is obtained by taking the codifferential of the tangent vector $\sigma:=\sigma^{1} \frac{\partial}{\partial x^{1}}+\sigma^{2} \frac{\partial}{\partial x^{2}}$ after first lowering its indices.)

The multisymplectic $(1,2)$-form is

$$
\omega=-\mathrm{d}_{\mathrm{v}} q \wedge \star \mathrm{d}_{\mathrm{v}} p+\mathrm{d}_{\mathrm{v}} p \wedge \star \mathrm{d}_{\mathrm{v}} r .
$$

In coordinates,

$$
\omega=\sqrt{g} \mathrm{~d}_{\mathrm{v}} q \wedge\left(\mathrm{d}_{\mathrm{v}} p^{2} \wedge \mathrm{d} x^{1}-\mathrm{d}_{\mathrm{v}} p^{1} \wedge \mathrm{d} x^{2}\right)+\left(\mathrm{d}_{\mathrm{v}} p_{1} \wedge \mathrm{d} x^{1}+\mathrm{d}_{\mathrm{v}} p_{2} \wedge \mathrm{d} x^{2}\right) \wedge \mathrm{d}_{\mathrm{v}} r_{12} .
$$

Thus the vector field of vertically closed $(0,2)$-forms $\boldsymbol{\kappa}$ has the components

$$
\kappa^{1}=-\sqrt{g} \mathrm{~d}_{\mathrm{v}} q \wedge \mathrm{d}_{\mathrm{v}} p^{1}-\mathrm{d}_{\mathrm{v}} p_{2} \wedge \mathrm{d}_{\mathrm{v}} r_{12}, \quad \kappa^{2}=-\sqrt{g} \mathrm{~d}_{\mathrm{v}} q \wedge \mathrm{d}_{\mathrm{v}} p^{2}+\mathrm{d}_{\mathrm{v}} p_{1} \wedge \mathrm{d}_{\mathrm{v}} r_{12} .
$$

\subsection{Example: Fluid kinematics via the TEA bundle}

Consider the kinematics of an incompressible fluid on a stationary three-dimensional Riemannian manifold $X$. Working in local coordinates, a velocity field $\mathbf{u}=u^{i} \partial / \partial x^{i}$ corresponds to the one-form $v=u_{i} \mathrm{~d} x^{i} \in \Omega^{(1,0)}(X)$, as described in [1]. Assuming that the fluid density is constant, the incompressibility condition amounts to

$$
\delta_{\mathrm{h}} v=0 .
$$

Now apply the Hodge decomposition to $v$ :

$$
v=\mathrm{d}_{\mathrm{h}} \phi+\delta_{\mathrm{h}} \Psi+v_{\Delta} .
$$


Here $\phi \in \Omega^{(0,0)}(X), \quad \Psi \in \Omega^{(2,0)}(X)$, and $v_{\Delta} \in \Omega^{(1,0)}(X)$ is a harmonic one-form, so that

$$
\mathrm{d}_{\mathrm{h}} v_{\Delta}=\delta_{\mathrm{h}} v_{\Delta}=0 .
$$

(If $X$ is compact, the harmonic $k$-forms are in 1:1 correspondence with the de Rham cohomology classes $H^{k}(X)$; in particular, if $H^{1}(X)=\{0\}$ then $v_{\Delta}=0$.) In the fluid mechanics literature the analogous decomposition applied to $\mathbf{u}$ is called the Helmholtz decomposition. The twoform $\Psi$ corresponds to a vector potential, which can be chosen to be solenoidal without loss of generality ( $\S 6.4$ of MARshalL [20]). Adopting this convention, we apply the constraint

$$
\mathrm{d}_{\mathrm{h}} \Psi=0 .
$$

Another important two-form corresponds to the vorticity, namely

$$
\omega=\mathrm{d}_{\mathrm{h}} v
$$

To represent the above system on the TEA bundle, we use the volume form to recast the equations as follows:

$$
\left[\begin{array}{cccc}
0 & \delta_{\mathrm{h}} & 0 & 0 \\
\mathrm{~d}_{\mathrm{h}} & 0 & \delta_{\mathrm{h}} & 0 \\
0 & \mathrm{~d}_{\mathrm{h}} & 0 & \delta_{\mathrm{h}} \\
0 & 0 & \mathrm{~d}_{\mathrm{h}} & 0
\end{array}\right]\left(\begin{array}{c}
\phi \\
v \\
\Psi \\
\text { vol }
\end{array}\right)=\left(\begin{array}{c}
0 \\
v-v_{\Delta} \\
\omega \\
0
\end{array}\right)
$$

In this formulation $v_{\Delta}$ and $\omega$ are considered to be given. So given the vorticity, inverting the operator on the left gives the velocity field, so it is a geometric formulation of the Biot-Savart law. The left-hand side of (5.14) is a multisymplectic Dirac operator, and the right hand side is the differential of

$$
H \mathrm{vol}=\frac{1}{2} v \wedge \star v+\star \Psi \wedge \boldsymbol{\omega}-v_{\Delta} \wedge \star v
$$

For two-dimensional flows, the reduced system

$$
\left[\begin{array}{ccc}
0 & \delta_{\mathrm{h}} & 0 \\
\mathrm{~d}_{\mathrm{h}} & 0 & \delta_{\mathrm{h}} \\
0 & \mathrm{~d}_{\mathrm{h}} & 0
\end{array}\right]\left(\begin{array}{c}
\phi \\
v \\
\Psi
\end{array}\right)=\left(\begin{array}{c}
0 \\
v-v_{\Delta} \\
\omega
\end{array}\right)
$$

applies; it is no longer necessary to specify that $d_{h} \Psi=0$, as this holds because of the reduction in dimension.

The above approach is of particular interest for flows that are computed on compact domains. For example, when periodic boundary conditions are imposed on flow in a rectangular or cubic domain, the cohomology is that of a (flat) torus. Consequently, the harmonic terms are significant. By contrast, the sphere has $H^{1}\left(S^{2}\right)=\{0\}$, so $v_{\Delta}=0$. One can also deal with moving domains $X$ (such as the atmosphere or ocean [11]) by replacing the second and third equations in (5.14) by equations for the absolute velocity and absolute vorticity. Then vol should be replaced by $f$ vol, where $f$ represents a potential for the part of the absolute vorticity that is due to the motion of $X$. 


\subsection{Construction of conservation laws}

We now use the multimomentum map defined in $\S 4$ to construct some simple conservation laws, using symmetries that arise in symplectic pattern formation [7]. The multisymplectic structure

$$
\omega=\sum_{k=1}^{n}(-1)^{k} \mathrm{~d}_{\mathrm{v}} u^{(k-1)} \wedge \star \mathrm{d}_{\mathrm{v}} u^{(k)}
$$

is invariant under the $2^{n}$-parameter Lie group of translations of the coefficients of $u^{(k)}$, for $k=0, \ldots, n$. These symmetries are generated by $\mathbf{v}_{\xi}=c^{i} \frac{\partial}{\partial u^{i}}$ and so

$$
\begin{aligned}
\left.\mathbf{v}_{\xi}\right\lrcorner \omega & \left.\left.=\sum_{k=1}^{n}\left\{(-1)^{k}\left(\mathbf{v}_{\xi}\right\lrcorner \mathrm{d}_{\mathrm{v}} u^{(k-1)}\right) \wedge \star \mathrm{d}_{\mathrm{v}} u^{(k)}+\mathrm{d}_{\mathrm{v}} u^{(k-1)} \wedge \star\left(\mathbf{v}_{\xi}\right\lrcorner \mathrm{d}_{\mathrm{v}} u^{(k)}\right)\right\} \\
& \left.\left.=\mathrm{d}_{\mathrm{v}} \sum_{k=1}^{n}\left\{-\left(\mathbf{v}_{\xi}\right\lrcorner \mathrm{d}_{\mathrm{v}} u^{(k-1)}\right) \wedge \star u^{(k)}+u^{(k-1)} \wedge \star\left(\mathbf{v}_{\xi}\right\lrcorner \mathrm{d}_{\mathrm{v}} u^{(k)}\right)\right\} .
\end{aligned}
$$

So action of the multimomentum map on the element $\mathbf{c} \in \mathfrak{g}$ is

$$
\left.\left.J(x, u)(\mathbf{c})=\lambda_{\xi}=c^{i} \sum_{k=1}^{n}\left\{-\left(\frac{\partial}{\partial u^{i}}\right\lrcorner \mathrm{d}_{\mathrm{v}} u^{(k-1)}\right) \wedge \star u^{(k)}+u^{(k-1)} \wedge \star\left(\frac{\partial}{\partial u^{i}}\right\lrcorner \mathrm{d}_{\mathrm{v}} u^{(k)}\right)\right\} .
$$

For instance, if $X=\mathbb{R}^{2}$ and $u=\left(u^{1}, u^{2}, u^{3}, u^{4}\right)=\left(q, p_{1}, p_{2}, r_{12}\right)$ then

$$
\begin{aligned}
\lambda_{\xi}= & -c^{1}\left(p_{1} \mathrm{~d} x^{2}-p_{2} \mathrm{~d} x^{1}\right)+c^{2}\left(q \mathrm{~d} x^{2}-r_{12} \mathrm{~d} x^{1}\right) \\
& -c^{3}\left(q \mathrm{~d} x^{1}+r_{12} \mathrm{~d} x^{2}\right)+c^{4}\left(p_{1} \mathrm{~d} x^{1}+p_{2} \mathrm{~d} x^{2}\right) .
\end{aligned}
$$

As $\lambda_{\xi}, L_{\alpha}^{i}$ and $g$ do not depend explicitly on $x^{1}$ or $x^{2}$, the condition (4.18) is satisfied provided that $H$ is invariant under a subgroup of the above translations. For example, if $H$ is independent of $p_{1}$ then there is a conservation law

$$
D_{1}(q)+D_{2}\left(r_{12}\right)=0
$$

As another example, consider the action of the group $\mathrm{SO}(2)$ on the TEA bundle above that has the generator

$$
\mathbf{v}_{\xi}=-p_{1} \frac{\partial}{\partial q}+q \frac{\partial}{\partial p_{1}}+r_{12} \frac{\partial}{\partial p_{2}}-p_{2} \frac{\partial}{\partial r_{12}}
$$

A short calculation yields

$$
\lambda_{\xi}=-\left(q r_{12}+p_{1} p_{2}\right) \mathrm{d} x^{1}+\frac{1}{2}\left(q^{2}+p_{1}^{2}-p_{2}^{2}-r_{12}^{2}\right) \mathrm{d} x^{2},
$$

Hence, when $H$ is invariant under this group action, the conservation law is

$$
D_{1}\left(\frac{1}{2}\left(q^{2}+p_{1}^{2}-p_{2}^{2}-r_{12}^{2}\right)\right)+D_{2}\left(q r_{12}+p_{1} p_{2}\right)=0 .
$$

Other conservation laws may be constructed similarly. 


\subsection{Lie point transformations admitted by the TEA bundle}

In this section, we examine which Lie point transformations are compatible with the multisymplectic structure $\omega$ on the TEA bundle. In other words, we answer the question: which point transformations lead to a multimomentum map? For Hamiltonian ODEs with $X=\mathbb{R}$ and $\omega=\mathrm{d}_{\mathrm{v}} p \wedge \mathrm{d}_{\mathrm{v}} q$, the symmetry generators

$$
\mathbf{v}_{\xi}=Q_{q} \frac{\partial}{\partial q}+Q_{p} \frac{\partial}{\partial p}
$$

are related to the momentum map $\lambda_{\xi}$ by

$$
\left.\mathbf{v}_{\xi}\right\lrcorner \omega=\mathrm{d}_{\mathrm{v}} \lambda_{\xi}
$$

In components,

$$
Q_{q}=-\frac{\partial \lambda_{\xi}}{\partial p}, \quad Q_{p}=\frac{\partial \lambda_{\xi}}{\partial q}
$$

Similarly, for multisymplectic PDEs, one can split the condition

$$
\left.\mathbf{v}_{\xi}\right\lrcorner \omega=\mathrm{d}_{\mathrm{v}} \lambda_{\xi}
$$

into $n 2^{n}$ components.

For instance, if $X=\mathbb{R}^{2}$ and $\left(u^{1}, u^{2}, u^{3}, u^{4}\right)=\left(q, p_{1}, p_{2}, r_{12}\right)$ as before, every point symmetry generator is of the form

$$
\mathbf{v}_{\xi}=Q^{1} \frac{\partial}{\partial q}+Q^{2} \frac{\partial}{\partial p_{1}}+Q^{3} \frac{\partial}{\partial p_{2}}+Q^{4} \frac{\partial}{\partial r_{12}}
$$

where $Q^{\alpha}=Q^{\alpha}(x, u)$. So

$$
\begin{aligned}
\left.\mathbf{v}_{\xi}\right\lrcorner \omega= & -Q^{1}\left(\mathrm{~d}_{\mathrm{v}} p_{1} \wedge \mathrm{d} x^{2}-\mathrm{d}_{\mathrm{v}} p_{2} \wedge \mathrm{d} x^{1}\right)+Q^{2}\left(\mathrm{~d}_{\mathrm{v}} q \wedge \mathrm{d} x^{2}-\mathrm{d} r_{12} \wedge \mathrm{d} x^{1}\right) \\
& -Q^{3}\left(\mathrm{~d}_{\mathrm{v}} q \wedge \mathrm{d} x^{1}+\mathrm{d}_{\mathrm{v}} r_{12} \wedge \mathrm{d} x^{2}\right)+Q^{4}\left(\mathrm{~d}_{\mathrm{v}} p_{1} \wedge \mathrm{d} x^{1}+\mathrm{d}_{\mathrm{v}} p_{2} \wedge \mathrm{d} x^{2}\right) .
\end{aligned}
$$

By equating this componentwise to

$$
\mathrm{d}_{\mathrm{v}} \lambda_{\xi}=\mathrm{d}_{\mathrm{v}}\left(\lambda_{\xi}^{1} \mathrm{~d} x^{2}-\lambda_{\xi}^{2} \mathrm{~d} x^{1}\right),
$$

we obtain

$$
Q^{1}=-\frac{\partial \lambda_{\xi}^{1}}{\partial p_{1}}=-\frac{\partial \lambda_{\xi}^{2}}{\partial p_{2}}, \quad Q^{2}=\frac{\partial \lambda_{\xi}^{1}}{\partial q}=\frac{\partial \lambda_{\xi}^{2}}{\partial r_{12}}, \quad Q^{3}=-\frac{\partial \lambda_{\xi}^{1}}{\partial r_{12}}=\frac{\partial \lambda_{\xi}^{2}}{\partial q}, \quad Q^{4}=\frac{\partial \lambda_{\xi}^{1}}{\partial p_{2}}=-\frac{\partial \lambda_{\xi}^{2}}{\partial p_{1}} .
$$

These constitute four first-order differential constraints on $\lambda_{\xi}$ (in contrast to the ODE case, where $\lambda_{\xi}$ is unconstrained). Provided that the above partial derivatives are continuous, we conclude that $\Phi=\lambda_{\xi}^{1}+i \lambda_{\xi}^{2}$ is a complex analytic function of $v=p_{1}+i p_{2}$ and of $w=q+i r_{12}$. The remaining condition (4.18) on $H$ and $\lambda_{\xi}^{i}$ enables one to find which symmetries are actually admitted by a given system of multisymplectic PDEs. Let $z=x^{1}+i x^{2}$; if we regard $\Phi$ as a function of $v, w, z$ and $\bar{z}$, the condition (4.18) amounts to

$$
\Re\left\{\frac{\partial \Phi}{\partial z}+\frac{\partial H}{\partial v} \frac{\partial \Phi}{\partial w}-\frac{\partial H}{\partial w} \frac{\partial \Phi}{\partial v}\right\}=0 .
$$


More generally, we can repeat the above process for any base space $X$. For convenience, we now use multi-index notation, denoting $k$-forms (with $k \geq 1$ ) on the TEA bundle by

$$
u_{\underline{\mathbf{J}}} \mathrm{d} x^{\mathbf{J}}=u_{j_{1} \cdots j_{k}} \mathrm{~d} x^{j_{1}} \wedge \cdots \wedge \mathrm{d} x^{j_{k}}, \quad j_{1}<\cdots<j_{k} .
$$

The symbol $\rightarrow$ denotes that the implied sum is to be taken over ascending indices only ${ }^{2}$. For consistency with (5.17), we denote the 0-form component of the TEA bundle by $u_{\emptyset}$.

It is useful to define $\mathrm{d} x^{\overrightarrow{\mathbf{J}}}$ to be the wedge product of all $\mathrm{d} x^{i}$ such that $i \notin \mathbf{J}$ (in ascending order). So in $\mathbb{R}^{3}$,

$$
\mathrm{d} x^{\overrightarrow{1}}=\mathrm{d} x^{2} \wedge \mathrm{d} x^{3} .
$$

Finally, let $|\mathbf{J}|$ denote the number of indices in $\mathbf{J}$. Then

$$
\begin{aligned}
\omega & =\sum_{l=1}^{n}(-1)^{l}\left(\sum_{|J|=l-1} \mathrm{~d}_{\mathfrak{v}} u_{\underline{\mathbf{J}}} \wedge \mathrm{d} x^{\mathbf{J}}\right) \wedge\left(\sum_{|\mathbf{K}|=l} \sqrt{g} \epsilon_{\underline{\mathbf{K}} \overrightarrow{\mathbf{K}}} \mathrm{d}_{\mathbf{v}} u^{\mathbf{K}} \wedge \mathrm{d} x^{\overrightarrow{\mathbf{K}}}\right) \\
& =\sum_{l=1}^{n} \sum_{|J|=l-1} \sum_{|\mathbf{K}|=l} \sqrt{g} \epsilon_{\underline{\mathbf{K}} \overrightarrow{\mathbf{K}}} \mathrm{d}_{\mathbf{v}} u^{\mathbf{K}} \wedge \mathrm{d}_{\mathbf{v}} u_{\underline{\mathbf{J}}} \wedge \mathrm{d} x^{\mathbf{J}} \wedge \mathrm{d} x^{\overrightarrow{\mathbf{K}}} .
\end{aligned}
$$

Here $\epsilon_{\underline{\mathbf{K}} \overrightarrow{\mathbf{K}}}$ is 1 (resp. -1 ) if $\underset{\underline{\mathbf{K}}}{\overrightarrow{\mathbf{K}}}$ is an even (resp. odd) permutation of $1,2, \cdots, n$. Also

$$
\mathrm{d}_{\mathrm{v}} u^{\mathrm{K}}=\mathrm{d}_{\mathrm{v}} u^{k_{1} \cdots k_{l}}=g^{k_{1} k_{1}^{\prime}} \cdots g^{k_{l} k_{l}^{\prime}} \mathrm{d}_{\mathrm{v}} u_{k_{1}^{\prime} \cdots k_{l}^{\prime}} .
$$

Note that the only contributions to the sum come from terms for which each index in $\mathbf{J}$ also belongs to $\mathbf{K}$. Therefore, with

$$
\mathbf{v}_{\xi}=Q_{\underline{\mathbf{J}}} \frac{\partial}{\partial u_{\mathbf{J}}}
$$

we obtain

$$
\left.\left.\mathbf{v}_{\xi}\right\lrcorner \omega=\sum_{i=1}^{n} \sum_{\mathbf{J} \not i i}(-1)^{\nu(\mathbf{J}, i)}\left(Q^{i \underline{\mathbf{J}}} \mathrm{d}_{\mathbf{v}} u_{\underline{\mathbf{J}}}-Q_{\underline{\mathbf{J}}} \mathrm{d}_{\mathrm{v}} u \stackrel{i \mathbf{J}}{\stackrel{\mathbf{J}}{ }}\right) \wedge\left(D_{i}\right\lrcorner \mathrm{vol}\right),
$$

where $\nu(\mathbf{J}, i)$ is the number of indices in $\mathbf{J}$ whose value is $\leq i$. Consequently (5.16) can be split into $n 2^{n}$ components by comparing coefficients of $\mathrm{d}_{\mathbf{v}} u_{\underline{\mathbf{K}}}$ in

$$
\mathrm{d}_{\mathrm{v}} \lambda_{\xi}^{i}=\sum_{\mathbf{J} \not \supset i}(-1)^{\nu(\mathbf{J}, i)}\left(Q^{i \underline{\mathbf{J}}} \mathrm{d}_{\mathrm{v}} u_{\underline{\mathbf{J}}}-Q_{\underline{\mathbf{J}}} \mathrm{d}_{\mathrm{v}} u^{i \underline{\mathbf{J}}}\right) .
$$

The $2^{n}$ unknowns $Q_{\underline{\mathbf{K}}}$ can be found in terms of first derivatives of $\lambda_{\xi}^{i}$ with respect to $u_{\underline{\mathbf{J}}}$; the remaining $(n-1) 2^{n}$ conditions are constraints upon the functions $\lambda_{\xi}^{i}$.

If $X=\mathbb{R}^{n}$, these constraints take a particularly simple form:

$$
Q_{\mathbf{J}}= \begin{cases}(-1)^{\nu(\mathbf{J} \backslash i, i)} \frac{\partial \lambda_{\xi}^{i}}{\partial u_{\mathbf{J} \backslash i}}, & \mathbf{J} \ni i ; \\ (-1)^{\nu(i \mathbf{J}, i)} \frac{\partial \lambda_{\xi}^{i}}{\partial u_{\underline{i \mathbf{J}}}}, & \mathbf{J} \not \supset i .\end{cases}
$$

\footnotetext{
${ }^{2}$ The notation $\underline{\mathbf{J}}$ is suggested in $\S 14.1$ of FRANKEL [12].
} 
For instance, for $X=\mathbb{R}^{3}$ we obtain

$$
\begin{aligned}
Q_{\emptyset} & =-\frac{\partial \lambda_{\xi}^{1}}{\partial u_{1}}=-\frac{\partial \lambda_{\xi}^{2}}{\partial u_{2}}=-\frac{\partial \lambda_{\xi}^{3}}{\partial u_{3}}, & Q_{1} & =\frac{\partial \lambda_{\xi}^{1}}{\partial u_{\emptyset}}=\frac{\partial \lambda_{\xi}^{2}}{\partial u_{12}}=\frac{\partial \lambda_{\xi}^{3}}{\partial u_{13}}, \\
Q_{2} & =-\frac{\partial \lambda_{\xi}^{1}}{\partial u_{12}}=\frac{\partial \lambda_{\xi}^{2}}{\partial u_{\emptyset}}=\frac{\partial \lambda_{\xi}^{3}}{\partial u_{23}}, & Q_{3} & =-\frac{\partial \lambda_{\xi}^{1}}{\partial u_{13}}=-\frac{\partial \lambda_{\xi}^{2}}{\partial u_{23}}=\frac{\partial \lambda_{\xi}^{3}}{\partial u_{\emptyset}}, \\
Q_{12} & =\frac{\partial \lambda_{\xi}^{1}}{\partial u_{2}}=-\frac{\partial \lambda_{\xi}^{2}}{\partial u_{1}}=-\frac{\partial \lambda_{\xi}^{3}}{\partial u_{123}}, & Q_{13} & =\frac{\partial \lambda_{\xi}^{1}}{\partial u_{3}}=\frac{\partial \lambda_{\xi}^{2}}{\partial u_{123}}=-\frac{\partial \lambda_{\xi}^{3}}{\partial u_{1}}, \\
Q_{23} & =-\frac{\partial \lambda_{\xi}^{1}}{\partial u_{123}}=\frac{\partial \lambda_{\xi}^{2}}{\partial u_{3}}=-\frac{\partial \lambda_{\xi}^{3}}{\partial u_{2}}, & Q_{123} & =\frac{\partial \lambda_{\xi}^{1}}{\partial u_{23}}=-\frac{\partial \lambda_{\xi}^{2}}{\partial u_{13}}=\frac{\partial \lambda_{\xi}^{3}}{\partial u_{12}} .
\end{aligned}
$$

\section{Concluding remarks}

The variational bicomplex provides a natural framework for multisymplectic PDEs. By separating horizontal and vertical derivatives, we have been able to generalize many of the key ideas from Hamiltonian ODEs. In particular, we have found the conditions that must be satisfied by a multimomentum map. The TEA bundle is a natural generalization of the cotangent bundle; it gives rise to a nondegenerate multisymplectic structure that extends the canonical symplectic two-form of Hamiltonian mechanics. These constructions give new insight into the structure of nonlinear multisymplectic PDEs.

\section{Acknowledgements}

This work was partially supported by EPSRC visiting fellowship grant GR/S58256/01, which funded a visit of JKL to the University of Surrey. JKL thanks Luca Sbano for helpful comments, and the Department of Mathematics at Surrey for their hospitality.

\section{References}

[1] V.I. Arnold \& B.A. Khesin. Topological Methods in Hydrodynamics, Appl. Math. Sciences 125, Springer-Verlag: New York (1998).

[2] R. Abraham and J.E. Marsden. Foundations of Mechanics, Second Edition, AddisonWesley: New York (1978).

[3] I.M. Anderson. The Variational Bicomplex, book manuscript; Utah State University (1989). http://www.math.usu.edu/ fg-mp/Publications/VB/vb.pdf

[4] I.M. Anderson. Introduction to the variational bicomplex. in Mathematical aspects of classical field theory, Contemp. Math. 132 51-73 (1992).

[5] A. Binz, J. Śniatycki, \& H. Fischer, Geometry of Classical Fields, North-Holland Mathematics Studies 154, North-Holland: Amsterdam, (1988). 
[6] T.J. Bridges. Multi-symplectic structures and wave propagation, Math. Proc. Camb. Phil. Soc. 121 147-190 (1997).

[7] T.J. BRIDGEs. Toral-equivariant partial differential equations and quasiperiodic patterns, Nonlinearity 11 467-500 (1998).

[8] T.J. Bridges. Canonical multi-symplectic structure on the total exterior algebra bundle, Proc. Roy. Soc. London A 462 1531-1551 (2006).

[9] T.J. Bridges \& F.E. LAIne-PEARson Multi-symplectic relative equilibria, multi-phase wavetrains and coupled NLS equations, Stud. Appl. Math. 107 137-155 (2001).

[10] F. Cantrijn, A. Ibort \& M. De LeÓn. On the geometry of multisymplectic manifolds, J. Austral. Math. Soc. (Ser. A) 66 303-330 (1999).

[11] M.J.P. Cullen, R.J. Douglas, I. Roulstone, \& M.J. Sewell. Generalized semigeostrophic theory on a sphere, J. Fluid Mech. 531 123-157 (2005).

[12] T. Frankel. The Geometry of Physics, Cambridge University Press (1997).

[13] M.J. Gotay, J. Isenberg, J.E. Mardden, \& R. Montgomery. Momentum maps and classical fields. Part I: Covariant field theory, arXiv preprint physics/9801019 (1998).

[14] P.E. Hydon. Multisymplectic conservation laws for differential and differential-difference equations, Proc. Royal Soc. London A 461 1627-1637 (2005).

[15] I. Kanatchikov. Canonical structure of classical field theory in the polymomentum phase space, Rep. Math. Phys. 41 49-90 (1998).

[16] J.K. Lawson. A frame-bundle generalization of multisymplectic geometry, Rep. Math. Phys. 45 183-205 (2000).

[17] J.K. LAWson. A frame-bundle generalization of multisymplectic momentum mappings, Rep. Math. Phys. 53 19-37 (2004).

[18] M. de León, M. McLean, L.K. Norris, A. Rey-Roca \& M. Salgado. Geometric structures in field theory, Preprint arXiv.org math-ph/0208036 (2002).

[19] J.E. Marsden \& T.S. Ratiu. Introduction to Mechanics and Symmetry, Texts Appl. Math. 17, Second edition, Springer-Verlag: New York (1999).

[20] J.S. Marshall. Inviscid Incompressible Flow, John Wiley \& Sons: New York (2001).

[21] L.K. NorRIS. Generalized symplectic geometry on the frame bundle of a manifold, Proc. Symp. Pure Math. 54, Amer. Math. Soc. Publ. 435-465 (1993).

[22] C. PAufler \& H. RÖMER. Geometry of Hamiltonian n-vector fields in multisymplectic field theory, J. Geom. Phys. 44 52-69 (2002). 
[23] D.J. Saunders. The Geometry of Jet Bundles, LMS Lecture Note Series 142, Cambridge University Press (1989).

[24] W.M. TulczyJEW. The Euler-Lagrange resolution, in Lecture Notes in Mathematics 836 , Springer-Verlag, New York (1980) pp. 22-48.

[25] A.M. Vinogradov. A spectral sequence associated with a non-linear differential equation, and the algebro-geometric foundations of Lagrangian field theory with constraints, Sov. Math. Dokl. 19 144-148 (1978).

[26] A.M. Vinogradov. The C-spectral sequence, Lagrangian formalism and conservation laws I, II, J. Math. Anal. Appl. 100 1-129 (1984). 